\title{
TNF Blockade, CNS Autoimmunity, Sex, and the Microbiome
}

\section{McKarns SC ${ }^{1,2 *}$ and Moisson FE}

${ }^{1}$ Department of Microbiology and Immunology, University of Missouri, Columbia, MO 65212, USA

${ }^{2}$ Laboratory of TGF- $\beta$ Biology, Epigenetics, and Cytokine Regulation, Center for Cellular and Molecular Immunology, Department of Surgery, University of Missouri School of Medicine, Columbia, MO 65212, USA

Keywords: TNFR2; CNS Demyelination; Autoimmunity; Gender; Sex; Microbiome

Tumor necrosis factor- $\alpha$ (TNF), a double-edged sword with both pro-inflammatory and anti-inflammatory properties, is one of the most widespread clinically targeted cytokines [1-4]. Anti-TNF therapies, such as infliximab, adalimumab, golimumab, and certolizumab, are central to the treatment of several autoimmune diseases, including rheumatoid arthritis (RA), inflammatory bowel disease (IBD), ankylosing spondylitis (SpA), and psoriasis. TNF blockade may also offer a potential avenue for the treatment of acute neuromyelitis optica (NMO) and/or NMO spectrum disorders (NMOSD) [5]. However, the use of currently available anti-TNF therapeutics is limited by their association with new onset or exacerbation of neuroinflammatory demyelinating disorders, including multiple sclerosis (MS), optic neuritis (ON), and acute transverse myelitis [2]. The precise mechanisms that predispose patients who receive anti-TNF treatment to benefit or increase risk of central nervous system (CNS) demyelination are not well-understood, but several theories have been proposed. First, TNF blocking agents may not penetrate the endothelial blood brain barrier (BBB), but rather precipitate disease by augmenting peripheral T cell auto-reactivity [6,7]. Second, TNF blockade may skew cytokine production, i.e., decrease IL-10 and increase IL-12 production, to facilitate demyelination [8]. Third, TNF blockers may "sponge" and thus lower the level of TNF in the periphery, thereby restricting disease alleviation to organs outside of the CNS. And fourth, reduced peripheral TNF levels may unmask a latent infection to propagate an autoimmune process [9]. The objective of this commentary is to bring to the forefront an alternative theory that highlights the influence of the gut microbiome on not only development but also treatment of autoimmune diseases [10].

The multiplicity of effects that TNF imparts on immunological responses, such as host defense, inflammation, cell death, and tissue repair, emanate from a two-ligand, two-receptor signaling system as well as differential expression of both ligands and their receptors. This pleotropic cytokine is produced mostly by monocytes and macrophages, but also lymphoid cells, microglia, astrocytes, dendritic cells, natural killer cells, and others. TNF is synthesized as a monomeric transmembrane molecule (tmTNF) and is cleaved from the cell surface by TNF converting enzyme (TACE) to release a soluble form of TNF (sTNF). Homotrimeric TNF (tmTNF and sTNF) binds to one of two distinct receptors, type 1 TNF receptor (TNFR1) or type 2 TNF receptor (TNFR2). TNFR1 is ubiquitously expressed and is activated in response to sTNF to promote inflammation, apoptosis, and demyelination. In contrast, TNFR2 expression is largely restricted to endothelial, hematopoietic, microglial, and some neuronal cells; has a higher affinity for tmTNF than sTNF; promotes cell survival; CD4 ${ }^{+}$Foxp ${ }^{+} \mathrm{T}$ regulatory (Treg) cell expansion; oligodendrocyte regeneration; and nerve remyelination [4,11-13]. Additionally, the gene for TNF is linked to the human major histocompatibility complex (MHC) located on chromosome 6 and TNFRSF1A, encoding TNFR1, is recognized as a risk allele for MS [14,15]. Animal models of experimental autoimmune encephalomyelitis (EAE) have associated TNFR1 and TNFR2 deficiency with decreased and increased disease severity, respectively, to suggest a selective role for TNFR1 in CNS demyelination. Thus, selective inhibition of sTNF/TNFR1 signaling, leaving beneficial tmTNF/ TNFR2 signaling intact may open new opportunities for TNF-selective next-generation therapeutics for the prevention and/or treatment of CNS autoimmune disorders (Figure 1).

Previous studies in mice have revealed that $\mathrm{CD} 4^{+} \mathrm{T}$ cell-intrinsic TNFR2 promotes Il2 expression [16]. Given that IL-2 is required for the expansion and function of $\mathrm{CD}^{+} \mathrm{FoxP}^{+} \mathrm{T}$ cells (Tregs), Miller et al., used a genetic loss-of-function approach to determine whether selective ablation of TNFR2 is sufficient to augment $\mathrm{MOG}_{35-55}$-specific $\mathrm{CD} 4^{+} \mathrm{T}$ cell auto-reactivity [10]. Surprisingly, 59 of $64(92 \%)$ of female, but only 5 of $60(8 \%)$ of male, C57BL/6J TNFR2 $2^{-/-} 2 \mathrm{D} 2$ Foxp3 $3^{\text {gfp }}$ reporter mice developed fulminant spontaneous autoimmune disease. A similar increase in spontaneous disease incidence was not observed in female TNFR2 $^{-1-}$ that did not carry the $2 \mathrm{D} 2 \mathrm{~T}$ cell receptor transgene. While a clear understanding of the underlying mechanisms remain elusive, these results are consistent with the view that anti-TNF therapy exacerbates risk in those patients who are already at risk (due to a genetic predisposition) for developing immune-mediated demyelination [17]. Augmented disease was also absent in $\mathrm{TNF}^{-/-} 2 \mathrm{D} 2$ mice to implicate distinct roles for TNFR1 and TNFR2. Histologically, lesions were absent from the brain, but the optic nerves and spinal cord exhibited extensive inflammation, demyelination, and axonal loss, with infiltration of predominantly B cells and T cells. With the noted exception of hightiter of MOG antibodies, the disease is highly reminiscent of NMOlike pathology. Importantly, it has yet to be determined whether the presence of MOG antibodies in TNFR2 ${ }^{-1-} 2 \mathrm{D} 2$ mice reflect an underlying pathogenic mechanism, a secondary immune response, a simple bystander phenomenon, or even a beneficial effect.

Strikingly, maternal antibiotic treatment protected TNFR2 ${ }^{-/} 2 \mathrm{D} 2$ offspring from developing spontaneous disease. The microbiome, consisting of the trillions of microorganisms (bacteria, viruses, and fungi) residing in our bodies, is a rapidly emerging area of interest in the medical community. While most microorganisms in our microbiome are beneficial or harmless, changes in the microbiome (dysbiosis) have been linked to diseases, including type 1 diabetes (T1D) and IBD $[18,19]$. To explore the possible connection between changes in the microbiome and female-biased spontaneous disease development in TNFR2 $2^{-1-}$ 2D2 mice, the Miller et al., first demonstrated that cross-fostering of TNFR2 $2^{--} 2 \mathrm{D} 2$ pups from the birth (donor) to recipient wild-type mothers, completely devoid of antibiotic treatment, restored disease susceptibility in female TNFR2 ${ }^{-1-} 2 \mathrm{D} 2$ mice. Data was then collected on the composition of gut microbiome in male and female $\mathrm{TNFR}^{-/-} 2 \mathrm{D} 2$ and TNFR2 $2^{-/-}$mice. $16 \mathrm{~S}$ rRNA gene amplicon sequencing of fecal samples identified a distinct gut microbiota profile, including a higher abundance of Akkermansia muciniphila, Sutterella

${ }^{*}$ Corresponding author: Susan C. McKarns, Department of Surgery, University of Missouri School of Medicine, M616 Medical Sciences Building, Columbia, MO 65212. E-mail: mckarnss@health.missouri.edu

Received April 25, 2016; Accepted May 03, 2016; Published May 10, 2016

Citation: McKarns SC, Moisson FE (2016) TNF Blockade, CNS Autoimmunity, Sex, and the Microbiome. J Mult Scler (Foster City) 3:172. doi:10.4172/23760389.1000172

Copyright: (c) 2016 McKarns SC, et al. This is an open-access article distributed under the terms of the Creative Commons Attribution License, which permits unrestricted use, distribution, and reproduction in any medium, provided the original author and source are credited. 


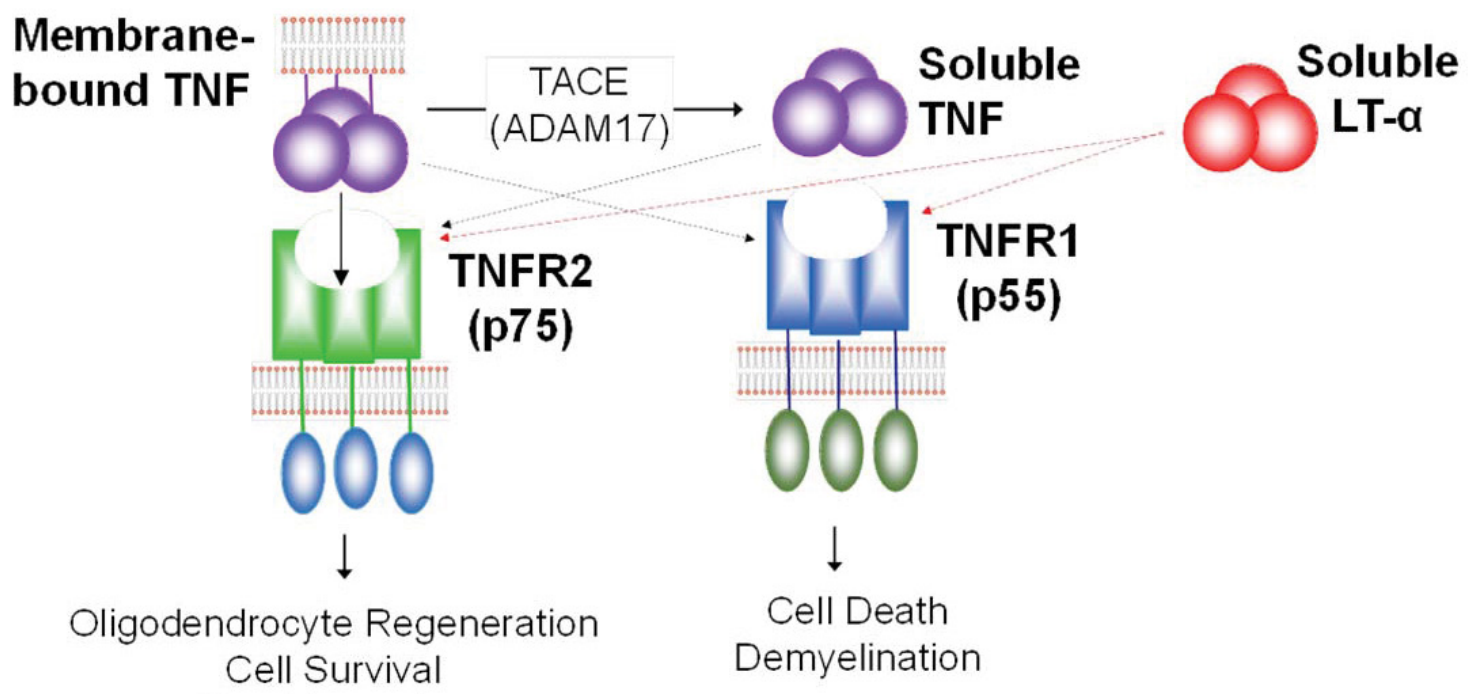

Treg Expansion

Remyelination

Figure 1: Shedding is an important for the control of TNFR1 and TNFR2 activity.

sp., Oscillospira sp., Bacteroides acidifaciens, and Anaeroplasma sp. in male TNFR2 ${ }^{-1-} 2 \mathrm{D} 2$ mice that associated with disease protection. These results strongly implicate that interactions between environmental (e.g., TNFR2 signaling blockade and sex hormones) and genetic factors (e.g., 2D2 auto-reactivity) with gut microbiota contribute to the development of spontaneous disease in female $\mathrm{TNFR}^{-/-} 2 \mathrm{D} 2$ mice. Importantly, these observations highlight the importance of the microbiome on not only development but also treatment of autoimmune disease. Malebiased microbiome-mediated protection has also been implicated in disease progression in the NOD T1D mouse model [20]. Collectively, the TNFR2 ${ }^{--}$2D2 model raises an interesting question of whether antiTNF treatment may be linked to myelin-specific or cross-reactivity to environmental myelin-similar peptides (commensal bacteria), in AQP4-IgG seronegative, MOG-IgG seropositive NMO and/or NMO spectrum disorder patients [21-23].

In conclusion, experimental models and clinical trials suggest a role for TNF blockade in CNS demyelination. The potential for sTNF and tmTNF to exert different functions in different cells under normal and pathological conditions within the CNS has warranted investigations to delineate the distinct functions of these two ligands. The report by Miller et al., identifying gut microbiota as a putative TNFR2-selective factor that affects autoimmune disease development in genetically susceptible animals, is consistent with the idea that selective targeting of TNFR1-mediated signaling, while sparing TNFR2 activation, may lessen adverse effects of anti-TNF therapies in the CNS. The findings in this report further suggest that investigations aimed to better understand distinct sTNF and tmTNF functions should be extended to include the intestines and the composition of commensal microbiota. Lastly, this report highlights that individuals may respond differently to anti-TNF therapy, in part, because of the commensal microbes that they carry, and further emphasizes the importance of sex and gender when studying mechanisms by which TNF blockade may affect health and disease processes.

(Figure 1). Shedding is an important for the control of TNFR1 and TNFR2 activity. There are two forms of tumor necrosis factor- $\alpha$ (TNF), a membrane-bound protein (tmTNF) and a soluble form (sTNF).
sTNF is generated by cleavage of tmTNF by the metalloproteinase, TACE (TNF converting enzyme), alternatively called ADAM 17 (adisintegrin andmetalloproteinase), from the cell membrane. TNF mediates its pleoptropic functions through two distinct receptors. Type 1 TNF receptor (TNFR1) expression is ubiquitous and largely constitutive. In contrast, type 2 TNF receptor (TNFR2) expression is more restricted to cells of the immune system, such as B and T lymphocytes (especially $\mathrm{CD}^{+}$FoxP3 $^{+} \mathrm{T}$ regulatory cells) , macrophages, but also epithelial cells of the gut, microglia, and neurons. Like tmTNF, TNFR1 and TNFR2 are shed from the cell surface by TACE/ADAM17 and released into the extracellular compartment. tmTNF bind and signals through TNFR2 with higher affinity than TNFR1; whereas, sTNF has a higher affinity for TNFR1 than TNFR2. Soluble Lymphotoxin-a (LT- $\alpha$ ), also called TNF- $\beta$, binds TNFR1 and TNFR2 with comparable affinity.

\section{References}

1. Probert $L$ (2015) TNF and its receptors in the CNS: The essential, the desirable and the deleterious effects. below Neuroscience 302: 2-22.

2. Kaltsonoudis E, Voulgari PV, Konitsiotis S, Drosos AA (2014) Demyelination and other neurological adverse events after anti-TNF therapy. below Autoimmun Rev 13: 54-58.

3. McCoy MK, Tansey MG (2008) TNF signaling inhibition in the CNS implications for normal brain function and neurodegenerative disease. below J Neuroinflammation 5: 45.

4. Steinman L, Merrill JT, McInnes IB, Peakman M (2012) Optimization of current and future therapy for autoimmune diseases. below Nat Med 18: 59-65.

5. Zhang H, Bennett JL, Verkman AS (2011) Ex vivo spinal cord slice model of neuromyelitis optica reveals novel immunopathogenic mechanisms. below Ann Neurol 70: 943-954.

6. Pardridge WM (1998) CNS drug design based on principles of blood-brain barrier transport. below J Neurochem 70: 1781-1792.

7. Robinson WH, Genovese M C, Moreland L W (2001) Demyelinating and neurologic events reported in association with tumor necrosis factor alpha antagonism: by what mechanisms could tumor necrosis factor alpha antagonists improve rheumatoid arthritis but exacerbate multiple sclerosis? Arthritis Rheum 44: 1977-1983.

8. van Boxel-Dezaire $A H$, Hoff SC, van Oosten BW, Verweij $C L$, Drager A M (1999) Decreased interleukin-10 and increased interleukin-12p40 mRNA are associated with disease activity and characterize different disease stages in multiple sclerosis. Ann Neurol 45: 695-703. 
Citation: McKarns SC, Moisson FE (2016) TNF Blockade, CNS Autoimmunity, Sex, and the Microbiome. J Mult Scler (Foster City) 3:172. doi:10.4172/2376-0389.1000172

Page 3 of 3

9. Mikuls TR, Weaver AL (2003) Lessons learned in the use of tumor necrosis factor-alpha inhibitors in the treatment of rheumatoid arthritis. Curr Rheumatol Rep 5: 270-277.

10. Miller PG, Bonn M B, Franklin CL, Ericsson AC, McKarns SC (2015) TNFR2 Deficiency Acts in Concert with Gut Microbiota To Precipitate Spontaneous Sex-Biased Central Nervous System Demyelinating Autoimmune Disease. J Immunol 195: 4668-4684.

11. Faustman D, Davis M (2010) TNF receptor 2 pathway: drug target for autoimmune diseases. below Nat Rev Drug Discov 9: 482-493.

12. Chen X, Bäumel M, Männel DN, Howard OM, Oppenheim JJ (2007) Interaction of TNF with TNF receptor type 2 promotes expansion and function of mouse CD4+CD25+ T regulatory cells. below J Immunol 179: 154-161.

13. Arnett HA, Mason J, Marino M, Suzuki K, Matsushima GK, et al. (2001) TNF alpha promotes proliferation of oligodendrocyte progenitors and remyelination. below Nat Neurosci 4: 1116-1122.

14. Spies T, Morton CC, Nedospasov SA, Fiers W, Pious D, et al. (1986) Genes for the tumour necrosis factors alpha and beta are linked to the human major histocompatibility complex. Proc Natl Acad Sci USA 83: 8699-8702.

15. Gregory AP, Dendrou CA, Attfield KE, Haghikia A, Xifara DK, et al. (2012) TNF receptor 1 genetic risk mirrors outcome of anti-TNF therapy in multiple sclerosis. Nature 488: 508-511.

16. Miller PG, Bonn MB, McKarns SC2 (2015) Transmembrane TNF-TNFR2
Impairs Th17 Differentiation by Promoting II2 Expression. below J Immunol 195: 2633-2647.

17. Nielsen NM, Westergaard T, Rostgaard K, Frisch M, Hjalgrim H, et al. (2005) Familial risk of multiple sclerosis: a nationwide cohort study. below Am J Epidemiol 162: 774-778.

18. Kostic AD, Gevers D, Siljander H, Vatanen T, Hyotylainen T, et al. (2015) The dynamics of the human infant gut microbiome in development and in progression toward type 1 diabetes. Cell Host Microbe 17: 260-273.

19. Ray K (2016) Gut microbiota: Dysbiosis in fungal microbiota in IBD. below Nat Rev Gastroenterol Hepatol 13: 188.

20. Markle JG, Frank DN, Adeli K, von Bergen M, Danska JS (2014) Microbiome manipulation modifies sex-specific risk for autoimmunity. below Gut Microbes 5: 485-493.

21. Berer K, Mues M, Koutrolos M, Rasbi ZA, Boziki M, et al. (2011) Commensa microbiota and myelin autoantigen cooperate to trigger autoimmune demyelination. Nature 479: 538-541.

22. Krishnamoorthy G, Saxena A, Mars LT, Domingues HS, Mentele R, et al. (2009) Myelin-specific $T$ cells also recognize neuronal autoantigen in a transgenic mouse model of multiple sclerosis. below Nat Med 15: 626-632.

23. Melamed E, Levy M, Waters PJ, Sato DK, Bennett JL, et al. (2015) Update on biomarkers in neuromyelitis optica. Neurol Neuroimmunol Neuroinflamm 2 e134
Citation: McKarns SC, Moisson FE (2016) TNF Blockade, CNS Autoimmunity, Sex, and the Microbiome. J Mult Scler (Foster City) 3:172. doi:10.4172/23760389.1000172
OMICS International: Publication Benefits \& Features Unique features:

- Increased global visibility of articles through worldwide distribution and indexing

- Showcasing recent research output in a timely and updated manner

- Special issues on the current trends of scientific research

Special features:

- $700+$ Open Access Journals

$50,000+$ editorial team

Rapid review process

Quality and quick editorial, review and publication processing

Indexing at major indexing services

- Sharing Option: Social Networking Enabled

- Authors, Reviewers and Editors rewarded with online Scientific Credits

- Better discount for your subsequent articles

Submit your manuscript at: http://www.omicsonline.org/submission/ 